\title{
Prostatic Arterial Supply: Anatomic and Imaging Findings Relevant for Selective Arterial Embolization
}

\author{
Tiago Bilhim, MD, PhD, João Martins Pisco, MD, PhD, \\ Hugo Rio Tinto, MD, Lúcia Fernandes, MD, \\ Luís Campos Pinheiro, MD, PhD, Andrea Furtado, MD, Diogo Casal, MD, \\ Marisa Duarte, MD, José Pereira, MD, António G. Oliveira, MD, PhD, and \\ João E. G. O'Neill, MD, PhD
}

\begin{abstract}
Purpose: To describe the anatomy and imaging findings of the prostatic arteries (PAs) on multirow-detector pelvic computed tomographic (CT) angiography and digital subtraction angiography (DSA) before embolization for symptomatic benign prostatic hyperplasia (BPH).

Materials and Methods: In a retrospective study from May 2010 to June 2011, 75 men (150 pelvic sides) underwent pelvic CT angiography and selective pelvic DSA before PA embolization for BPH. Each pelvic side was evaluated regarding the number of independent PAs and their origin, trajectory, termination, and anastomoses with adjacent arteries.

Results: A total of $57 \%$ of pelvic sides $(n=86)$ had only one PA, and $43 \%(n=64)$ had two independent PAs identified (mean PA diameter, $1.6 \mathrm{~mm} \pm 0.3)$. PAs originated from the internal pudendal artery in $34.1 \%$ of pelvic sides $(\mathrm{n}=73)$, from a common trunk with the superior vesical artery in $20.1 \%(n=43)$, from the anterior common gluteal-pudendal trunk in $17.8 \%(n=38)$, from the obturator artery in $12.6 \%(n=27)$, and from a common trunk with rectal branches in $8.4 \%(n=18)$. In $57 \%$ of pelvic sides $(n=86)$, anastomoses to adjacent arteries were documented. There were 30 pelvic sides (20\%) with accessory pudendal arteries in close relationship with the PAs. No correlations were found between PA diameter and patient age, prostate volume, or prostate-specific antigen values on multivariate analysis with logistic regression.
\end{abstract}

Conclusions: PAs have highly variable origins between the left and right sides and between patients, and most frequently arise from the internal pudendal artery.

\section{ABBREVIATIONS}

$\mathrm{BPH}=$ benign prostatic hyperplasia, DSA = digital subtraction angiography, IMA = inferior mesenteric artery, MIP $=$ maximum intensity projection, $\mathrm{PA}=$ prostatic artery, PAE = prostatic artery embolization, PSA = prostate-specific antigen, $3 \mathrm{D}=$ three-dimensional

It has been suggested that prostatic artery (PA) embolization (PAE) for symptomatic benign prostatic hyperpla-

From the Departments of Anatomy (T.B., A.F., D.C., J.E.G.O.), Radiology (T.B., H.R.T., L.F.), Urology (L.C.P.), and Biostatistics (A.G.O.), Faculdade de Ciências Médicas (FCM), Universidade Nova de Lisboa; Department of Interventional Radiology (T.B., J.M.P., H.R.T., L.F., M.D., J.P.), Hospital Saint Louis; and Urology Department (L.C.P.), Hospital São José, Centro Hospitalar de Lisboa Central, Lisbon, Portugal. Received February 22, 2012; final revision received July 20, 2012; accepted July 31, 2012. Address correspondence to T.B., Rua Luz Soriano, no. 182, 1200-249 Lisbon, Portugal; E-mail: tiagobilhim@hotmail.com

None of the authors have identified a conflict of interest.

(c) SIR, 2012

J Vasc Interv Radiol 2012; 23:1403-1415

http://dx.doi.org/10.1016/j.jvir.2012.07.028 sia $(\mathrm{BPH})$ may become a common treatment like uterine artery embolization for uterine leiomyomas (1). Animal studies in pigs and dogs have shown that PAE can induce prostatic volume reduction, with no related sexual dysfunction $(2,3)$.

Preliminary studies of PAE for symptomatic relief of lower urinary tract symptoms in patients with BPH have shown promising results (4-6). One of the most challenging aspects when performing PAE is to identify the PAs and differentiate them from the surrounding arteries.

Cadaveric studies have addressed the PA anatomy $(7,8)$, with the description of two different arterial systems to the prostate gland: the cranial or vesicoprostatic branch that runs between the bladder base and the prostate that has hypertrophied and supplied most of the central gland ade- 


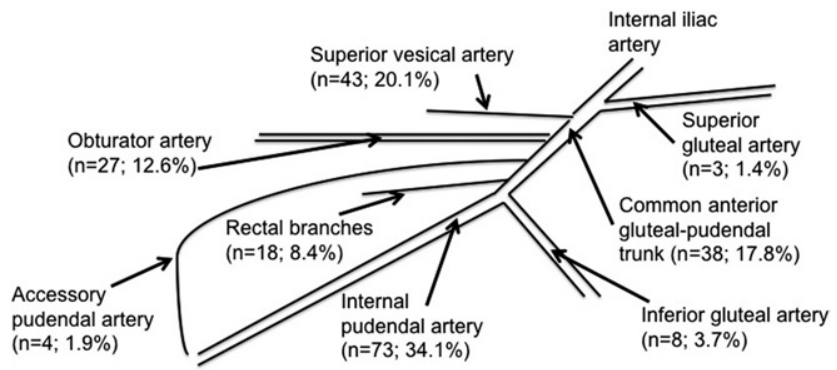

Figure 1. Schematic drawing of the different PA origins.

nomas in BPH, also called "artery of the adenoma"; and the caudal, which originates inferiorly, closer to the rectum (9). Also, more than one PA may be found in as many as $30 \%$ of pelvic sides (10). After reaching the prostate, the PAs have a corkscrew appearance $(7,8)$ and perforate the capsule in four quadrants, two anterior/lateral for the cranial PAs and two posterior/lateral for the caudal PAs (11-13).

Few studies have addressed radiologically the anatomy of the male pelvic arterial system $(14,15)$ and PAs $(16)$. Knowledge of the male pelvic and PA anatomy is needed to safely perform PAE. In this study, we report the main arterial variations in prostatic vascularization relevant for PAE by using multirow-detector pelvic computed tomographic (CT) angiography and digital subtraction angiography (DSA), based on the retrospective evaluation of 150 pelvic sides.

\section{MATERIALS AND METHODS}

\section{Patient Selection}

A retrospective study was conducted from May 2010 to June 2011 in 75 male patients (150 pelvic sides) who underwent pelvic CT angiography and selective pelvic DSA before PAE for BPH. Patient age ranged from 50 to 85 years, with a mean of 66.0 years. Institutional review board
Table 1. PA Origin and Arterial Anastomosis Type
Finding

PA origin

Internal pudendal artery

Superior vesical artery

Anterior common gluteal-pudendal trunk

Obturator artery

Prostatorectal trunk

Inferior gluteal artery

Accessory pudendal artery

Superior gluteal artery

Type of anastomosis

Internal pudendal arteries

Contralateral PAs

Ipsilateral PAs

Rectal arteries

Vesical arteries

Lateral accessory pudendal arteries
Incidence

$214(100)$

$73(34.1)$

$43(20.1)$

38 (17.8)

27 (12.6)

$18(8.4)$

8 (3.7)

4 (1.9)

$3(1.4)$

$42(43.3)$

$17(17.6)$

$13(13.4)$

$14(14.4)$

$11(11.3)$

30 (20)
$\mathrm{PA}=$ prostatic artery

Values in parentheses are percentages.

approval was obtained for the study, and all participants signed an informed consent form for PAE. All patients were informed about the embolization technique used, and the experimental nature was clearly included.

Included were male patients with a diagnosis of BPH with moderate to severe lower urinary tract symptoms refractory to medical treatment for at least 6 months or experiencing acute urinary retention. Patients were excluded in the case of malignancy, which was evaluated by prostate-specific antigen (PSA), physical examination, transrectal ultrasound (in all patients), prostatic biopsy (in suspicious cases), and advanced atherosclerosis/tortuosity of the iliac arteries or PAs evaluated by pelvic CT angiography performed before $\mathrm{PAE}$ in all

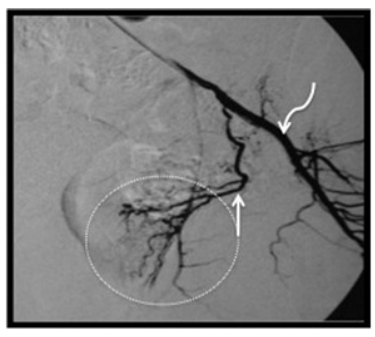

a.

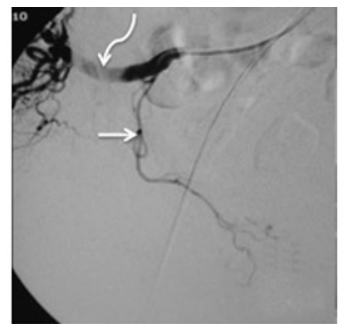

b.

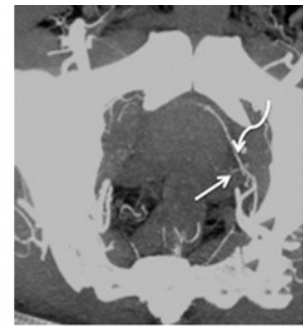

c.

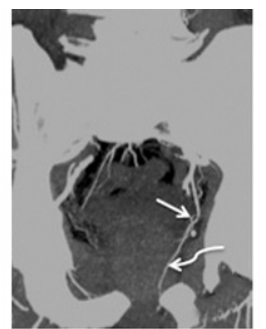

d.

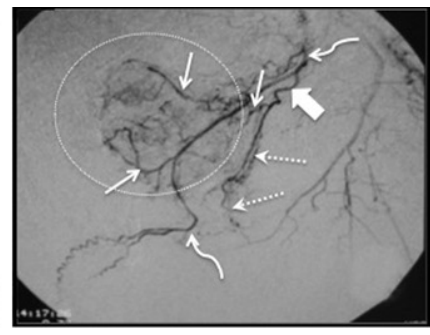

e.

Figure 2. Rare prostatic artery (PA) origins. (a) Digital subtraction angiography (DSA) with same-side anterior oblique projection ( $35^{\circ}$ ) and caudal/cranial angulation $\left(10^{\circ}\right)$ of a left-side PA (straight arrow) arising from the inferior gluteal artery (curved arrow). The dotted circle marks prostate gland opacification. (b) DSA with same-side anterior oblique projection $\left(35^{\circ}\right)$ and caudal/cranial angulation $\left(10^{\circ}\right)$ of a right-side PA (straight arrow) arising from the superior gluteal artery (curved arrow). (c) Computed tomographic (CT) angiography with axial maximum-intensity projection (MIP) reformat shows PA (straight arrow) arising from a lateral accessory pudendal artery (curved arrow). (d) CT angiography with coronal MIP reformat shows PA (straight arrow) arising from a lateral accessory pudendal artery (curved arrow). (e) Selective left-side DSA with same-side anterior oblique projection $\left(35^{\circ}\right)$ and caudal/cranial angulation $\left(10^{\circ}\right)$ shows two independent PAs (straight arrows) and one prostatorectal trunk (thick arrow) that bifurcates into the middle rectal artery (dotted arrows) and one small posterior/lateral PA (straight arrow) originating from the left-side lateral accessory pudendal artery, finishing as the dorsal artery of the penis (curved arrows). The dotted circle marks prostate gland opacification. 


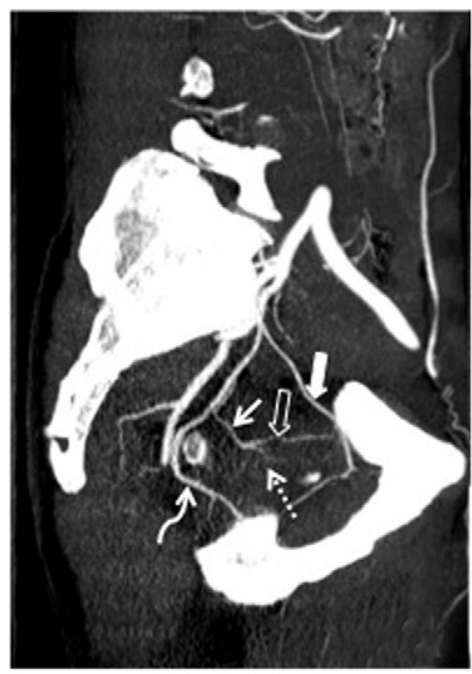

a.

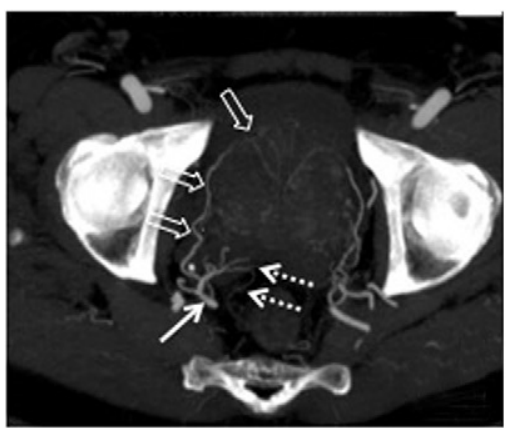

d.

b.
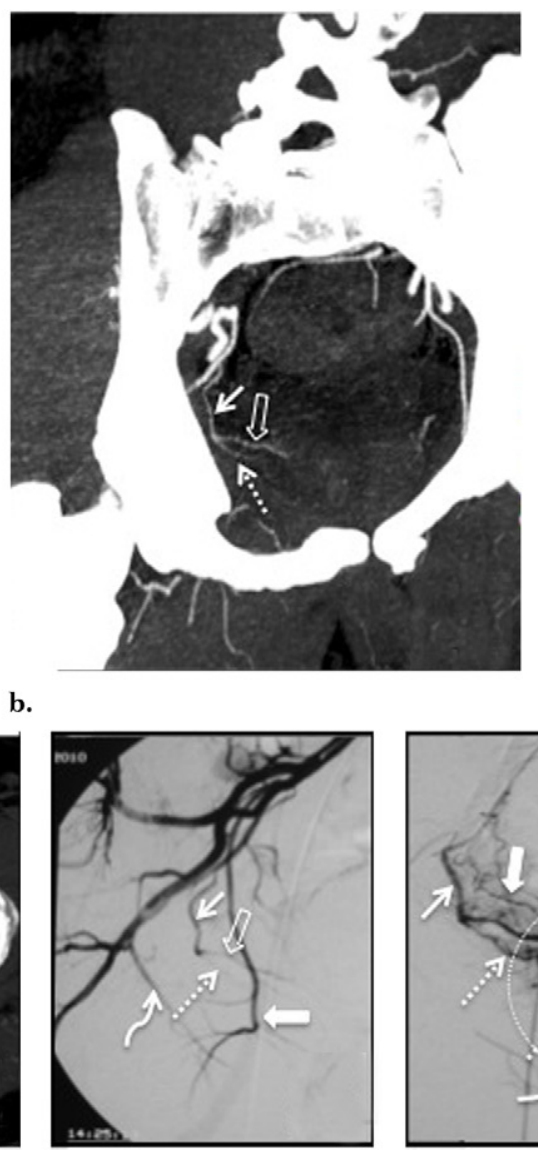

e.

f.

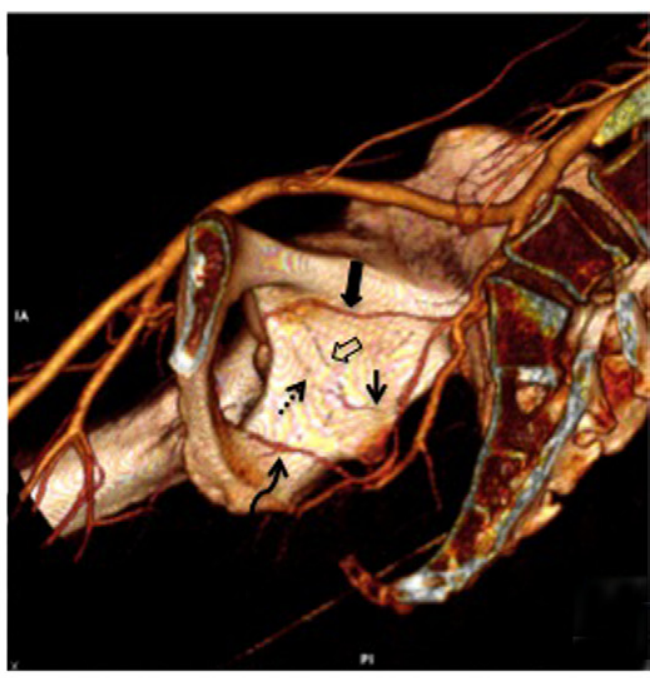

c.

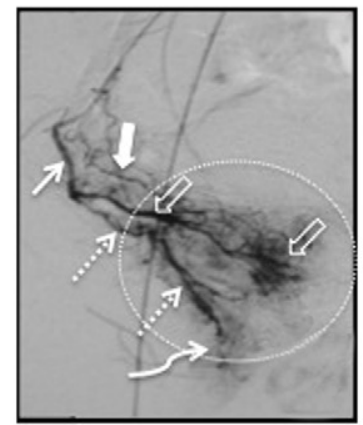

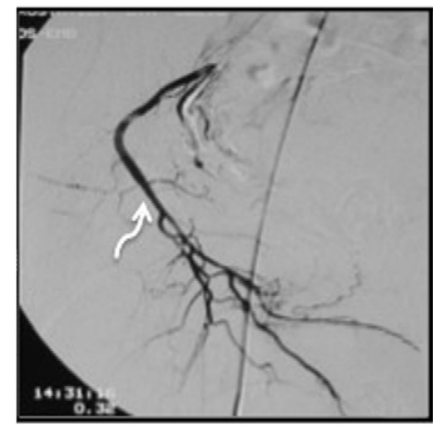

g.

Figure 3. Images from a case with a solitary PA. (a) Pelvic CT angiography with sagittal MIP reformat shows a solitary PA (straight arrow) arising from the internal pudendal artery (curved arrow) above the sciatic notch on the right pelvic side. After its origin, the PA bifurcates into two PAs: anterior/lateral, vascularizing the central gland (open arrows); and posterior/lateral, vascularizing the peripheral gland (dotted arrow). The solid arrow marks the obturator artery. (b) Pelvic CT angiography with coronal MIP reformat shows a solitary PA (straight arrow) that bifurcates into two PAs: anterior/lateral (open arrow) and posterior/lateral (dotted arrow). (c) Pelvic CT angiography with 3D reformat shows a solitary PA (straight arrow) arising from the internal pudendal artery (curved arrow). After its origin, the PA bifurcates into two PAs: anterior/lateral (open arrow), and posterior/lateral (dotted arrow). The solid arrow marks the obturator artery. (d) Pelvic CT angiography with axial MIP reformat shows a solitary PA (straight arrow) that bifurcates into two PAs: anterior/lateral, vascularizing the central gland (open arrows), and posterior/lateral, vascularizing the peripheral gland (dotted arrows). Note the small anastomoses of the posterior/lateral PA to rectal branches (dotted arrows). (e) DSA of the right internal iliac artery performed with same-side anterior oblique projection $\left(35^{\circ}\right)$ and caudal/cranial angulation $\left(10^{\circ}\right)$ shows a solitary PA (straight arrow) arising from the internal pudendal artery (curved arrow). After its origin, the PA bifurcates into two PAs: anterior/lateral (open arrow) and posterior/lateral (dotted arrow). The solid arrow marks the obturator artery. (f) DSA after selective catheterization of the right-side PA with same-side anterior oblique projection $\left(35^{\circ}\right)$ and caudal/cranial angulation $\left(10^{\circ}\right)$. A solitary PA (straight arrow) bifurcates into two PAs: anterior/lateral, vascularizing the central gland (open arrows), and posterior/lateral, vascularizing the peripheral gland (dotted arrows). The solid arrow marks the small anastomoses to the inferior vesical artery, the dotted circle marks prostate gland opacification, and the curved arrow marks the rectal/perineal branches of the posterior/lateral PA. (g) Control DSA with same-side anterior oblique projection $\left(35^{\circ}\right)$ and caudal/cranial angulation $\left(10^{\circ}\right)$ after selective PAE of the right-side PA. The curved arrow marks the internal pudendal artery. (Available in color online at www.jvir.org.)

patients. Only patients treated with bilateral PAE were included. Patients were excluded when bilateral selective PA anatomy characterization with CT angiography and DSA was not possible.

\section{Pelvic CT Angiography Protocol}

Pelvic CT angiography examinations were performed with a 16-slice spiral scanner (GE Medical Systems, Milwaukee, Wisconsin) in all patients in the supine position before embolization to exclude patients not suitable for treatment and to identify the branching pattern of the internal iliac artery and the arteries supplying the prostate. Power settings were $100-120 \mathrm{kV}$ and $200-300$ $\mathrm{mA}$, matrix of $512 \times 512$ pixels, collimation of $16 \times$ $1.25 \mathrm{~mm}$, slice thickness $1.25 \mathrm{~mm}$, and pitch of 1.3 . Iodinated contrast agent injection $(150 \mathrm{~mL}, 350 \mathrm{mg}$ $\mathrm{I} / \mathrm{mL}$, rate of $5 \mathrm{~mL} / \mathrm{s}$ ) was performed with bolus triggering in the abdominal aorta above the renal arteries. 


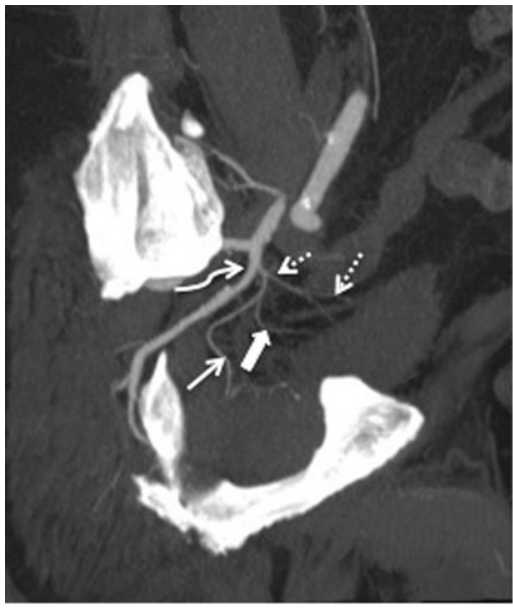

a.

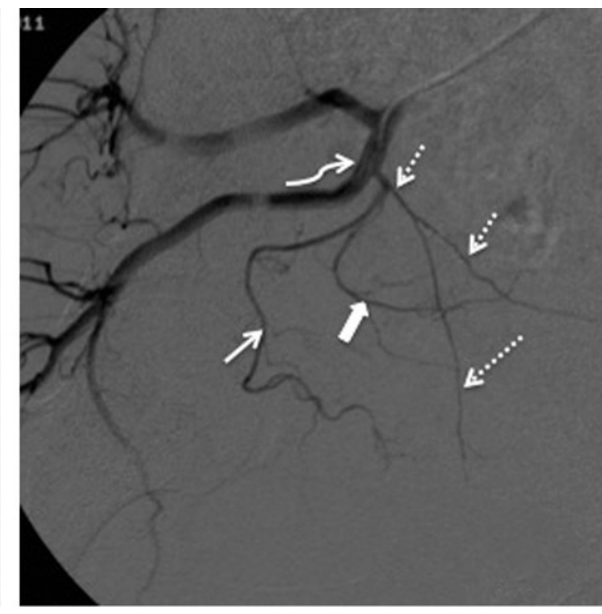

b.

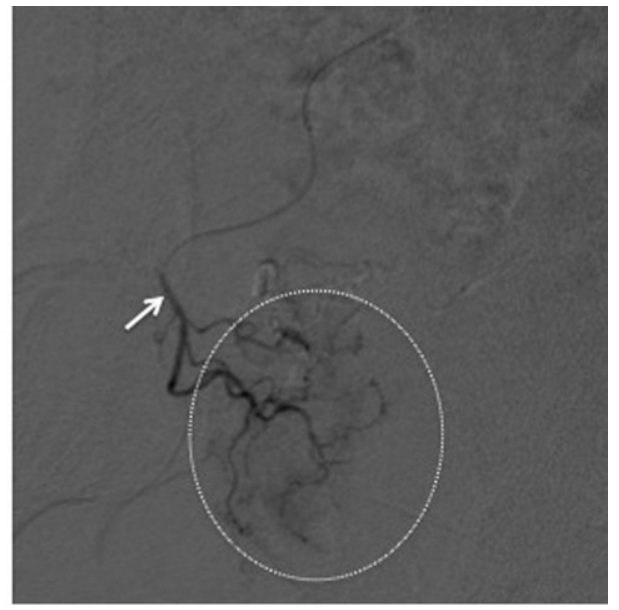

c.

Figure 4. On the right pelvic side, there is a solitary PA (straight arrow) originating from the superior vesical artery (dotted arrows). The inferior vesical artery is shown by the solid arrow. (a) Pelvic CT angiography with sagittal MIP reformat. The straight arrow marks solitary PA, dotted arrows mark the superior vesical artery, the curved arrow marks anterior common gluteal-pudendal trunk, and the solid arrow marks the inferior vesical artery. (b) DSA of right internal iliac artery with the catheter tip in the proximal part of the anterior division was performed with same-side anterior oblique projection $\left(35^{\circ}\right)$ and caudal $/$ cranial angulation $\left(10^{\circ}\right)$. The straight arrow marks solitary PA, dotted arrows mark the superior vesical artery, the curved arrow marks the anterior common gluteal/pudendal trunk, and the solid arrow marks the inferior vesical artery. (c) DSA after selective catheterization of the PA (arrow) with a microcatheter (the dotted circle marks prostate gland opacification).

Postprocessing with maximum-intensity projections (MIPs) and volume rendering with three-dimensional (3D) reformats was performed by one of the interventional radiologists who was present for all PAE procedures, with 5 years of experience in interventional radiology and CT angiography and 2 years of experience with PAE. Volumetric data was analyzed with $1.25 \mathrm{~mm}$ thick axial reformats with special attention given to the sagittal MIP and volume rendering with 3D reformats.

\section{DSA Protocol}

DSA was performed under local anesthesia in all patients via a unilateral (usually the right side) or bilateral femoral approach with a 5-F, 11-cm-long hydrophilic sheath (Cordis, Bridgewater, New Jersey). A Cobra-shaped catheter (C2F5; Cordis) or Roberts uterine artery catheter (Cook, Bloomington, Indiana) was introduced in the femoral artery to catheterize the contralateral hypogastric artery. The catheter tip was left in the proximal part of the anterior division, and DSA was performed at three frames per second $(3 \mathrm{~mL} / \mathrm{s})$ with $6 \mathrm{~mL}$ nonionic contrast medium (Ioversol $350 \mathrm{mg}$ $\mathrm{I} / \mathrm{mL}$; Covidien, Dublin, Ireland) with a same-side anterior oblique projection $\left(35^{\circ}\right)$ and caudal/cranial angulation $\left(10^{\circ}\right)$. The findings of DSA, 3D volume rendering, and sagittal MIP reformats of pelvic CT angiography were correlated regarding the number of independent PAs and their origin, direction, and termination. All PAs identified on CT angiography and seen on DSA were selectively catheterized with a Roberts uterine artery catheter or $2.5-\mathrm{F}$ microcatheter (Cantata [Cook] or Progreat [Terumo, Tokyo, Japan]) and 0.016-inch guide wire (Sagitta [Cook] or Glidewire GT [Terumo]). Selective PA DSA was per- formed manually with $3-5 \mathrm{~mL}$ of contrast agent volume in neutral and same-side anterior oblique $\left(35^{\circ}\right)$ and caudal/ cranial angulation $\left(10^{\circ}\right)$ projections. The contralateral internal iliac artery (usually the right) was selectively catheterized after the catheter was reformed into a Waltman loop. Selective PAE was performed with 100 - or 200- $\mu$ m nonspherical polyvinyl alcohol particles (Cook).

\section{PA Classification}

All PAs identified were classified according to their origin, direction, number of pedicles, termination with intraprostatic branches, corkscrew appearance of capsular branches, and anastomoses with surrounding arteries. Mean PA diameter was measured by CT angiography and selective prostatic DSA. Important anatomic variations relevant for PAE, such as lateral accessory pudendal arteries, were identified and characterized. To evaluate any possible relationships among patient age, prostate volume (log-transformed), PSA values (logtransformed), PA diameter, number of vascular pedicles, and presence of the corkscrew pattern, we performed univariate analyses with analyses of variance, Student $t$ test, and Fisher exact test as appropriate, and multivariate regression analyses. Stata software (release 12; Stata, College Station, Texas) was used for all analyses. Statistical differences were assumed with a $P$ value lower than .05 .

\section{RESULTS}

\section{Technical Results}

During the study period (May 2010 to June 2011), we evaluated 91 patients with pelvic CT angiography. We 


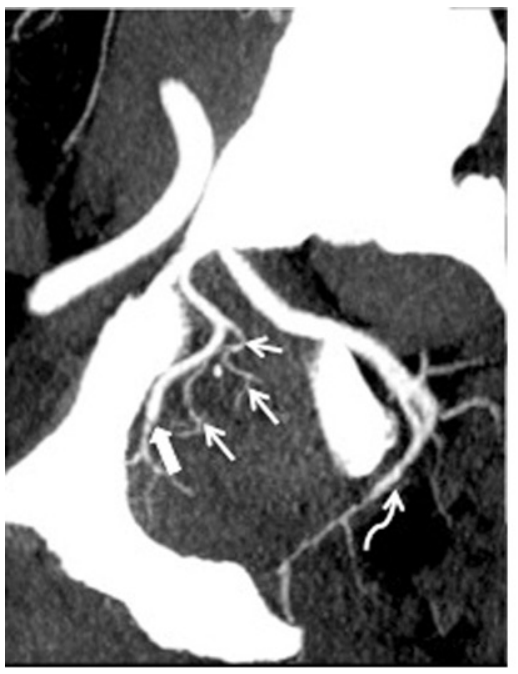

a.

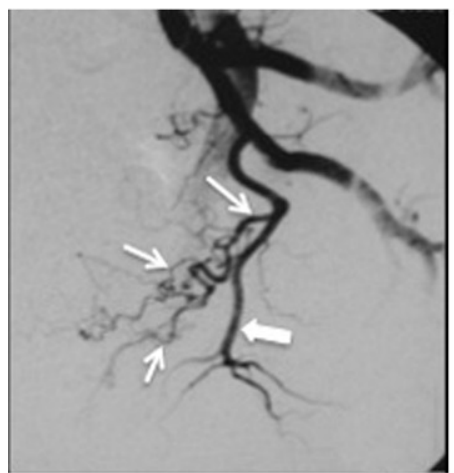

d. PA bifurcates into anterior/lateral and posterior/lateral PAs (straight arrows). (a) Pelvic CT angiography with sagittal MIP reformat. Straight arrows mark the PA, the solid arrow marks the obturator artery, and the curved arrow marks the internal pudendal artery. (b) Pelvic CT angiography with coronal MIP reformat. The straight arrow marks the PA and the solid arrow marks the obturator artery. (c) Pelvic CT angiography with three-dimensional (3D) reformat. Straight arrows mark the PA, the solid arrow marks the obturator artery, and the curved arrow marks the internal pudendal artery. (d) DSA of left internal iliac artery performed with same-side anterior oblique projection $\left(35^{\circ}\right)$ and caudal/cranial angulation $\left(10^{\circ}\right)$. Straight arrows mark the PA and the solid arrow marks the obturator artery. (e, f) DSA after selective catheterization of the left-side PA in neutral position before PAE. The straight arrow marks the PA and the solid arrow marks the obturator artery. (g) Control DSA in neutral position after PAE. The straight arrow marks the PA and the solid arrow marks the obturator artery. (Available in color online at www.jvir.org.)

excluded seven patients who underwent unilateral PAE $(8 \%)$ and two patients in whom selective PA catheterization was impossible bilaterally (2\%). Also, we excluded another seven patients $(8 \%)$ based on the evaluation of $\mathrm{CT}$ angiography alone as a result of tortuosity and atherosclerotic changes in the iliac arteries and PAs.

Therefore, PA anatomy as identified on CT angiography and DSA was compared in 75 patients (150 pelvic sides). Bilateral selective PA catheterization was possible in all patients with the use of a microcatheter in 52 patients $(69 \%)$ and a Roberts uterine artery catheter in the remaining 23 (31\%). Mean procedural DSA time was 86 minutes (range, 25-185 min) and mean fluoroscopic time was 27 minutes (range, 6-63 $\mathrm{min}$ ). Embolization was performed with a mean volume of $0.3 \mathrm{~mL}$ of polyvinyl alcohol, with $100-\mu \mathrm{m}$ particles in 32 patients $(43 \%)$ and $200-\mu \mathrm{m}$ particles in 43 patients $(57 \%)$.

\section{Number of Independent PAs and PA Origin}

Based on the retrospective analysis of pelvic CT angiography and selective DSA of the internal iliac arteries, it was possible to identify the number of independent PAs and their origin, trajectory, apparent capsular penetration, and termination. There was one PA in $57 \%$ of pelvic sides $(\mathrm{n}=$ $86)$ and two independent PAs in the other $43 \%(n=64)$, for a total of 214 PAs. Overall, we identified a mean of $2.9 \pm$ 0.9 independent PAs per patient (range, 2-4 PAs) with a mean diameter of $1.6 \mathrm{~mm} \pm 0.3$.

The most frequent PA origin was the middle third of the internal pudendal artery, above the sciatic notch $(34.1 \% ; \mathrm{n}=73)$. Other common origins were a common trunk with the superior vesical artery $(20.1 \% ; n=43)$, the anterior common gluteal/pudendal trunk $(17.8 \% ; \mathrm{n}=38)$, 

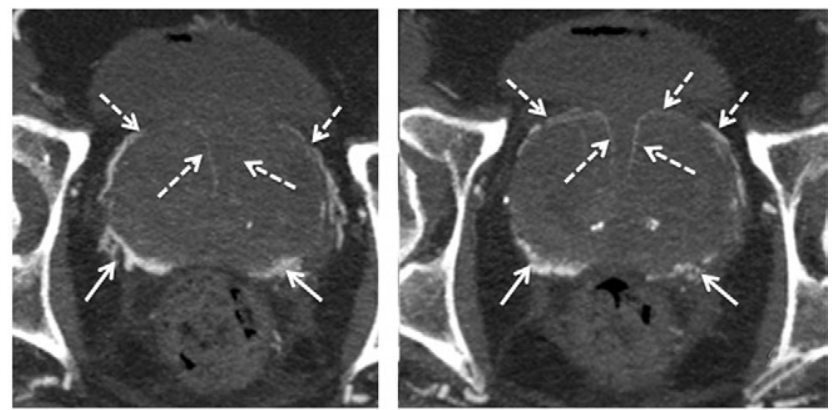

a.
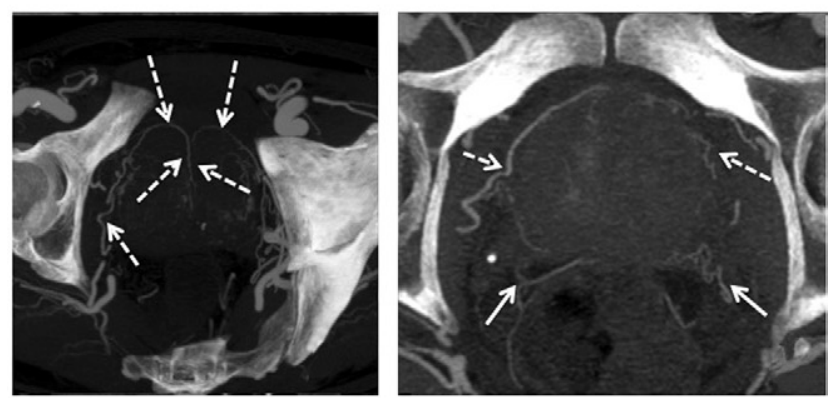

b.

c.
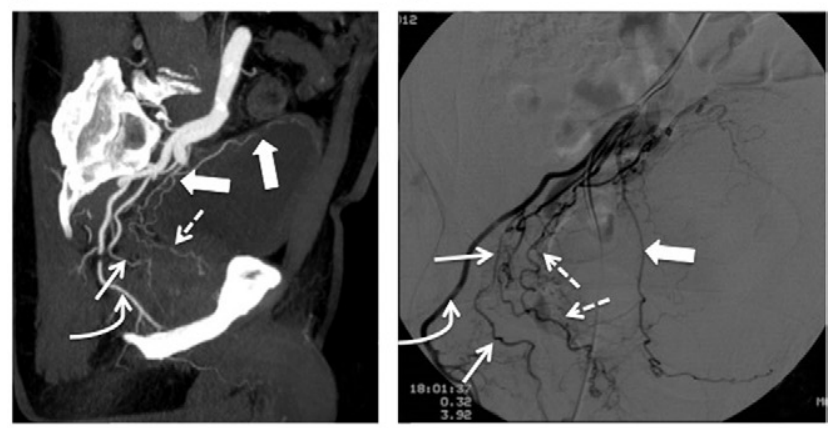

d.

e.

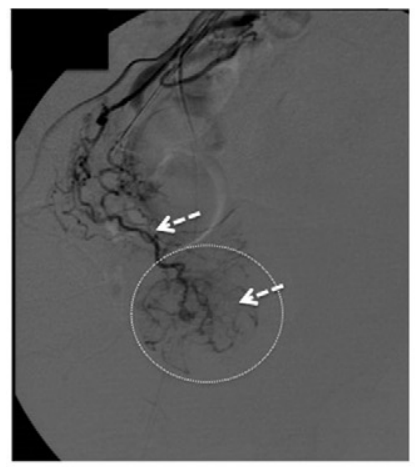

f.

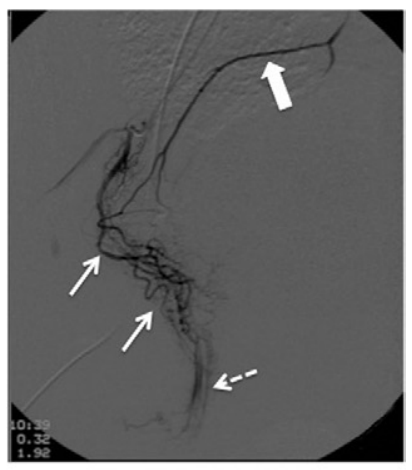

g.

Figure 6. Images from a case with two independent PAs. (a) Pelvic CT angiography with axial MIP shows that capsular branches penetrate the capsule in four quadrants: anterior/lateral PAs (dotted arrows mark central gland vascularization) and posterior/lateral PAs (solid arrows mark peripheral gland vascularization) on each pelvic side. The apparent capsular penetration of the anterior/lateral PAs (dotted arrows) is made approximately at the 2-o'clock and 10-o'clock positions for the left and right sides, respectively, and, for the posterior/lateral PAs, at the 5-o'clock and 7-o'clock positions for the left and right sides, respectively. Pelvic CT angiography with axial MIP shows (b) anterior/lateral PAs (dotted arrows) vascularizing most of the central gland and (c) anterior/lateral PAs (dotted arrows mark obturator artery $(12.6 \% ; \mathrm{n}=27)$, and a common trunk with rectal branches $(8.4 \% ; \mathrm{n}=18)$. Rare origins were the inferior gluteal artery $(3.7 \% ; \mathrm{n}=8)$, accessory pudendal artery $(1.9 \% ; \mathrm{n}=4)$, and superior gluteal artery $(1.4 \% ; \mathrm{n}=$ 3; Figs 1 and 2, Table 1).

\section{Solitary PAs}

Solitary PAs arose from the internal pudendal artery, superior vesical artery, common anterior gluteal/pudendal trunk, or obturator artery (Figs 3-5). Before reaching the prostatic capsule, at a variable distance from their origin, they bifurcated into two PAs, one anterior/lateral and the other posterior/lateral. The anterior/lateral PA followed an anterior and superior trajectory, penetrating the prostatic capsule at approximately the 2-o'clock and 10-o'clock positions for the left and right sides, respectively, vascularizing most of the central gland. The posterior/lateral PA had a more posterior and inferior trajectory, penetrating the prostatic capsule at approximately the 5-o'clock and 7-o'clock positions for the left and right sides, respectively, vascularizing most of the peripheral gland.

\section{Two Independent PAs}

When two independent PAs were identified, the anterior/ lateral PA had a more superior/proximal origin from the common anterior gluteal/pudendal trunk close to, or with a common origin with, the superior vesical artery, vascularizing most of the central gland. The posterior/lateral PA had an inferior/distal origin from the internal pudendal artery above the sciatic notch, vascularizing most of the peripheral gland (Figs 6 and 7). In these individuals, there was a close proximity between the vesical arteries and the anterior/

central gland vascularization) and posterior/lateral PAs (solid arrows mark peripheral gland vascularization) on each pelvic side. Note the close proximity of the posterior/lateral PAs with the rectum. (d-g) Two independent PAs on the right pelvic side. (d) Pelvic CT angiography with sagittal MIP reformat shows the anterior/lateral PA (dotted arrow) with a more superior/proximal origin from a short common trunk with the superior vesical artery (solid arrows). The posterior/lateral PA (straight arrow) has an inferior/ distal origin from the internal pudendal artery (curved arrow) above the sciatic notch. (e) DSA of the right internal iliac artery performed with same-side anterior oblique projection $\left(35^{\circ}\right)$ and caudal/cranial angulation $\left(10^{\circ}\right)$. Dotted arrows mark the anterior/lateral PA, the solid arrow marks the superior vesical artery, straight arrows mark the posterior/lateral PA, and the curved arrow marks the internal pudendal artery. (f) DSA with same-side anterior oblique projection $\left(35^{\circ}\right)$ and caudal/cranial angulation $\left(10^{\circ}\right)$ after selective catheterization of the anterior/lateral PA (dotted arrows). The dotted circle marks central gland opacification. (g) DSA with same-side anterior oblique projection $\left(35^{\circ}\right)$ and caudal/cranial angulation $\left(10^{\circ}\right)$ after selective catheterization of the posterior/lateral PA. Note the overlapping vascularization between the peripheral prostatic gland (arrows) and rectal branches (dotted arrow marks anal blush) with retrograde opacification of the IMA (solid arrow). 


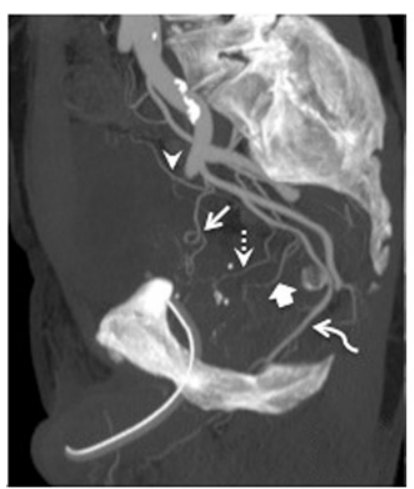

a.

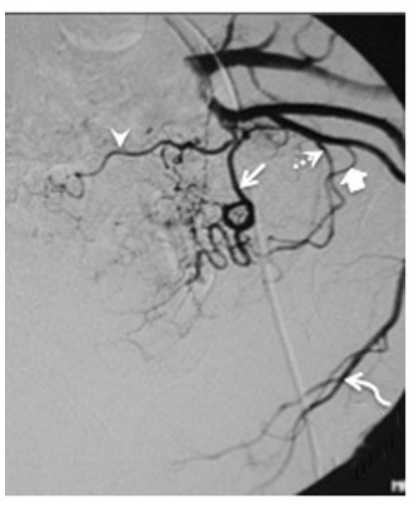

e.

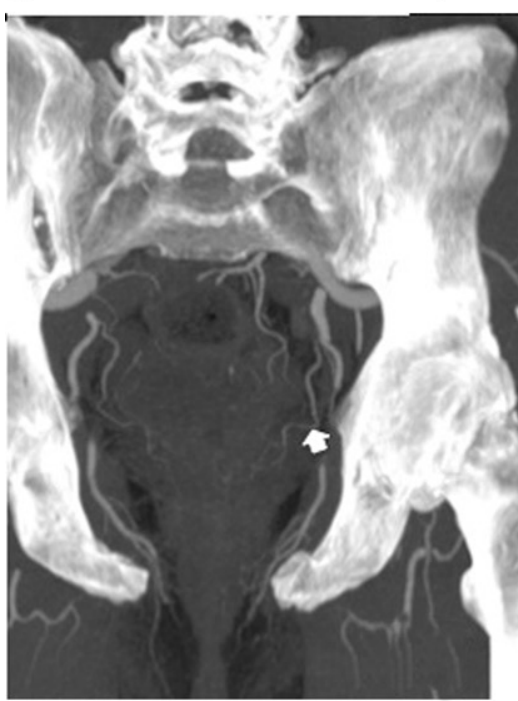

i.

b.

f.
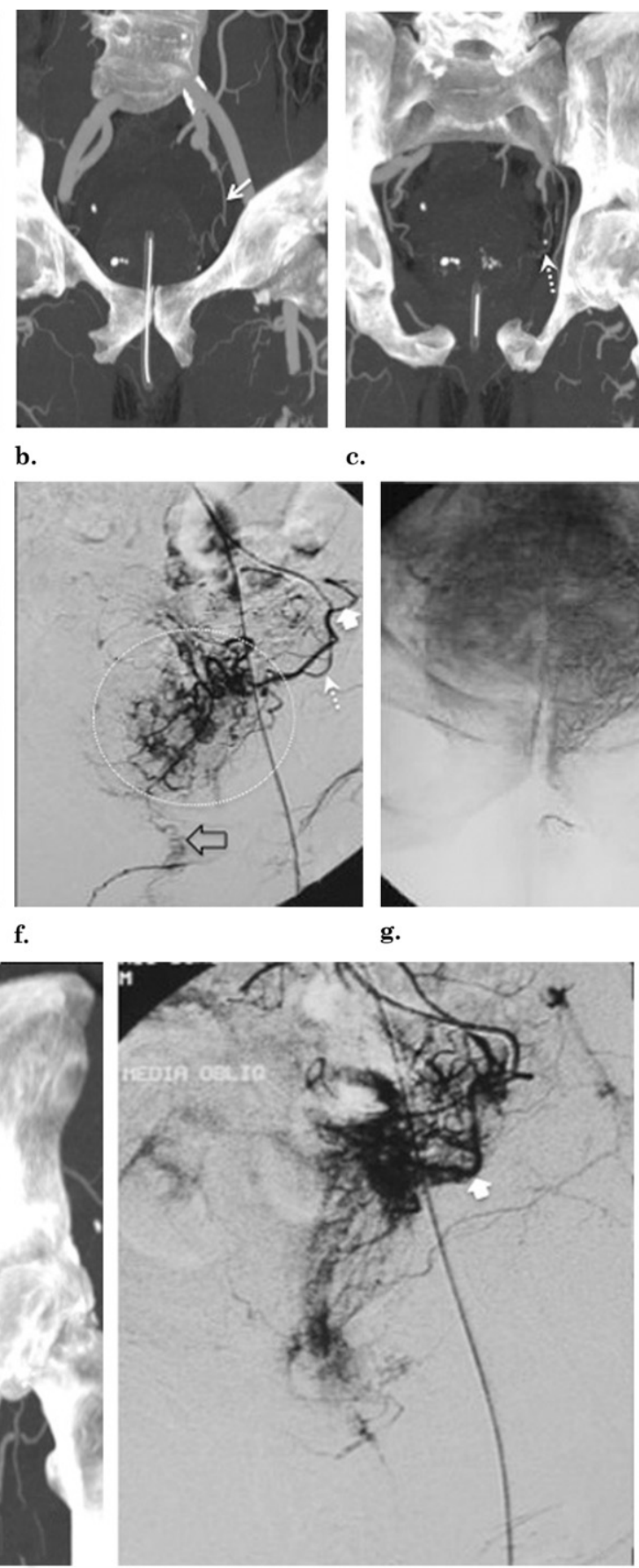

j.

c.

g.
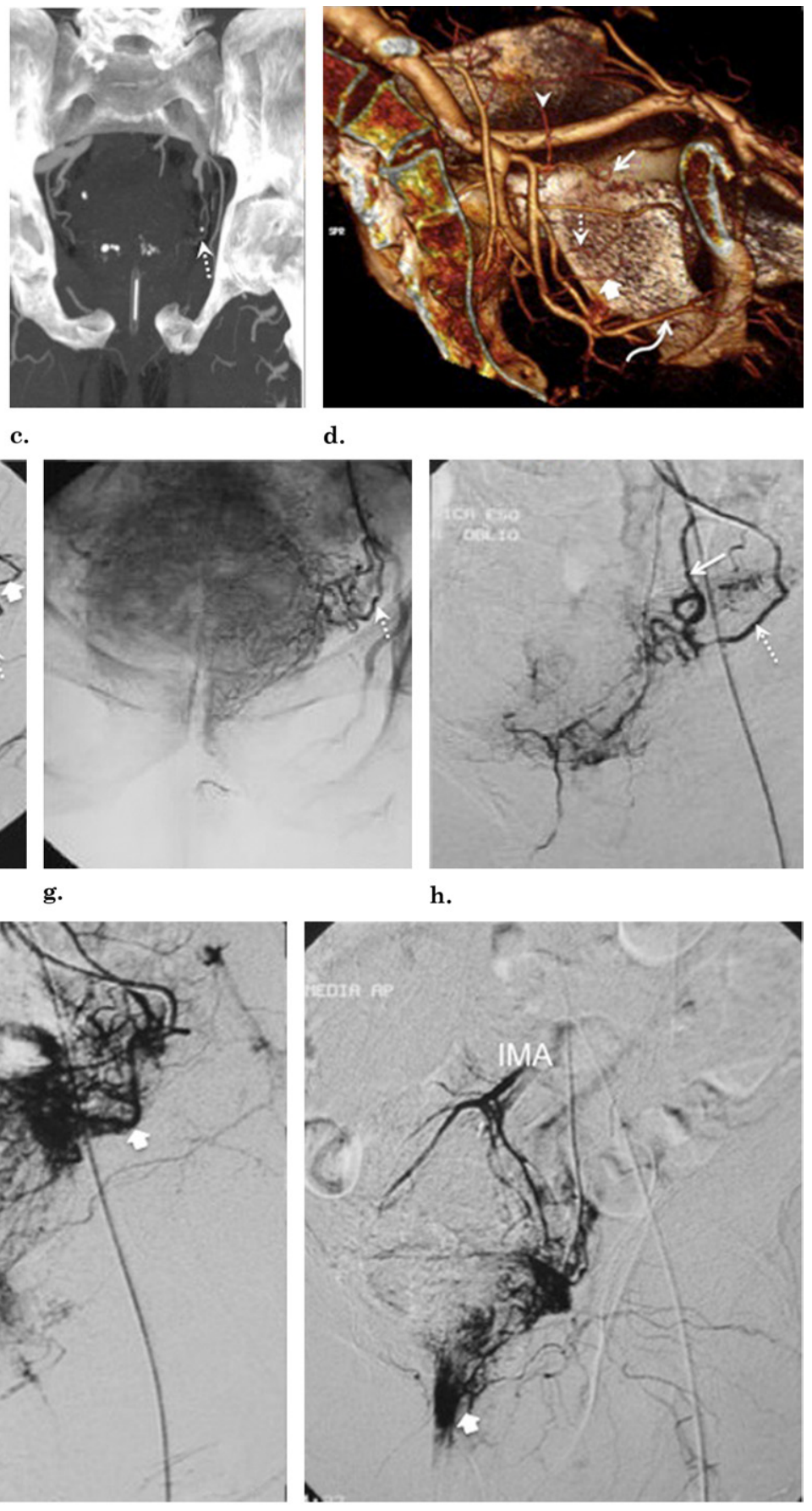

k.

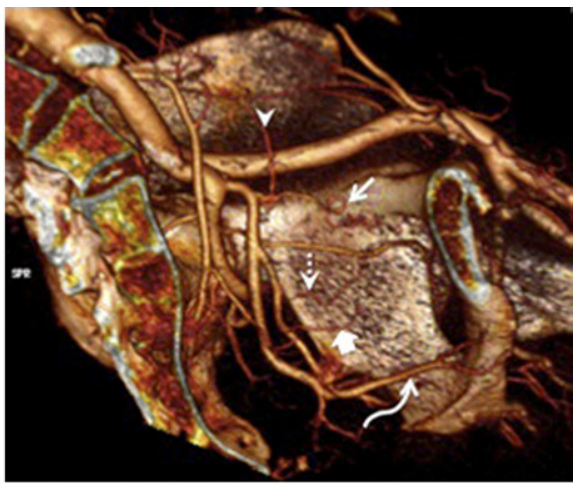

d.

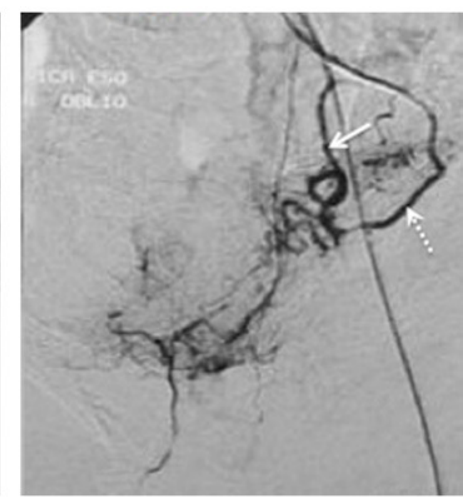

h.

Figure 7. Two independent PAs on the left pelvic side. (a) Pelvic CT angiography with sagittal MIP reformat. The anterior/lateral PA (straight arrow) has a more superior/proximal origin from a short common trunk with the superior vesical artery (arrowhead). The posterior/lateral PA (dotted arrow) has an inferior/distal origin from the internal pudendal artery (curved arrow) above the sciatic notch. Note the close proximity between the middle rectal artery (thick arrow) and the posterior/lateral PA (dotted arrow). (b) Pelvic CT angiography with coronal MIP reformat shows the anterior/lateral PA (arrow). (c) Pelvic CT angiography with coronal MIP reformat shows the posterior/lateral PA (arrow). (d) Pelvic CT angiography with 3D reformat. The straight arrow marks the anterior/lateral PA, the arrowhead marks the superior vesical artery, the dotted arrow marks the posterior/lateral PA, the curved arrow marks the internal pudendal artery, and the thick arrow marks the middle rectal artery. (e) DSA of internal iliac artery performed with same-side anterior oblique projection $\left(35^{\circ}\right)$ and caudal/cranial angulation $\left(10^{\circ}\right)$. The straight arrow marks the anterior/lateral PA, the arrowhead marks the superior vesical artery, the dotted arrow marks the posterior/lateral PA, the curved arrow marks the internal pudendal artery, and the thick arrow marks the middle rectal artery. (f) DSA with same-side anterior oblique projection $\left(35^{\circ}\right)$ and caudal/cranial angulation $\left(10^{\circ}\right)$ after selective catheterization of the posterior/lateral PA (dotted arrow). Note anastomoses with the rectal branches with retrograde opacification of the middle rectal artery (thick arrow), and anastomoses through small postcapsular branches to the internal pudendal artery (open arrow). Prostate gland opacification is seen inside the dotted circle. (g) DSA in neutral position after selective catheterization of the posterior/lateral PA (arrow) shows prostate gland opacification. (h) DSA with same-side anterior oblique projection (35 ) 
lateral PA and between the rectal arteries and the posterior/ lateral PA. It was easy to differentiate the anterior/lateral PA from the vesical arteries: vesical arteries had a straighter trajectory with a superior and more diffuse vascularization to the bladder wall, whereas the PAs had a downward undulating trajectory with a more globular and circumscribed vascularization below the bladder base. Differentiating the rectal arteries from the posterior/lateral PA was more difficult. The rectal arteries showed a more oblong and vertical opacification to the rectal wall with a prominent anal blush at the perineum, with retrograde filling of the inferior mesenteric artery (IMA). Posterior/lateral PAs had a more round and circumscribed appearance (Figs 8 and 9). These two circulations were frequently in close proximity, which made it impossible to differentiate and selectively catheterize them; on the contrary, the vesical circulation was easier to separate from the prostatic circulation by advancing the microcatheter distally to the vesical artery origin.

\section{Arterial Anastomoses}

Based on CT angiography and selective prostatic DSA, it was possible to evaluate the intraprostatic branches and anastomoses with surrounding arteries. No significant anastomoses were identified in $43 \%$ of pelvic sides $(n=64)$, and anastomoses to adjacent arteries were documented in 57\% $(\mathrm{n}=86)$. The most frequent types of anastomoses found were to the internal pudendal arteries $(43.3 \%)$, contralateral $(17.6 \%)$ and ipsilateral (13.4\%) prostatic branches, rectal arteries (14.4\%), and vesical arteries (11.3\%; Table 1). We found two different types of anastomoses relevant to the embolization technique: one through small pericapsular and postcapsular branches, the other through large precapsular branches that coursed alongside the anterior and lateral aspect of the prostate from the base to the apex, terminating in the perineum with anastomoses to the internal pudendal artery (also called lateral accessory pudendal arteries). Along their trajectory, these collateral vessels gave rise to capsular prostatic branches (Fig 10). There were 30 pelvic sides $(20 \%)$ with lateral accessory pudendal arteries representing anastomoses between the PAs and the internal pudendal arteries. We also found five pelvic sides (3.3\%) with lateral accessory pudendal arteries solely responsible for the arterial blood supply to the corpora cavernosa in close proximity with the PAs.

\section{PA DSA Findings}

The DSA findings of the PAs were very variable. A corkscrew pattern of the capsular branches was found in $37 \%$ of individuals $(\mathrm{n}=28)$. In the remaining patients, a globular circumscribed opacification was found below the bladder base, without remarkable features (Fig 10).

\section{Correlation between Anatomic and Clinical Data}

The number of PAs was correlated with the mean diameter of the PAs $(P<.0001)$; patients with only two PAs (one on each pelvic side) had a larger mean PA diameter $(1.9 \mathrm{~mm})$ than those with three $(1.5 \mathrm{~mm})$ or four $(1.3 \mathrm{~mm})$ PAs. The number of PAs did not correlate with age, prostate volume, PSA, or presence of the corkscrew pattern (Table 2). The same results were obtained with ordered logistic regression. The corkscrew pattern was associated with larger prostate volumes (mean, $111.9 \mathrm{~mL} ; P \leq .0001$ ) and higher PSA values $(8.1 \mathrm{ng} / \mathrm{mL} ; P=.004)$ compared with the absence of the corkscrew pattern (mean prostate volume, $67.0 \mathrm{~mL}$; mean PSA, $4.3 \mathrm{ng} / \mathrm{mL}$; Table 3). In multivariate analysis with logistic regression, only prostate volume was independently associated with the corkscrew pattern $(P=.01)$. We found no correlations between PA diameter and patient age, prostate volume, or PSA values.

\section{DISCUSSION}

We used CT angiography before DSA to exclude patients with advanced atherosclerosis/tortuosity of the iliac arteries or PAs ( $8 \%$ in the present study) and to allow us to draw a vascular map of the internal iliac arteries and PAs. PAs on DSA lack pathognomonic findings, and many small-sized arteries may easily be mistaken for PAs based only on DSA, leading to long procedural times and high radiation dose or nontarget embolization. The use of CT angiography before DSA reassures correct location before embolization, and we believe the use of the anterior oblique projection $\left(35^{\circ}\right)$ with caudal/cranial angulation $\left(10^{\circ}\right)$ in the initial DSA of the internal iliac artery is fundamental, with excellent correlation with the $\mathrm{CT}$ angiography sagittal MIP reformat, allowing easy recognition of the PAs and avoiding multiple DSA runs with different projections. The corkscrew pattern of the PAs may also help to identify the PAs on DSA: this was previously described in cadavers (7) and was present in $37 \%$ of individuals in the present study $(\mathrm{n}=28)$. As expected, this pattern was found in patients with larger prostates and higher PSA values, as it is probably easier to identify in these patients.

With preinterventional image guidance, PAE may be performed with a mean procedural time of approximately

and caudal/cranial angulation $\left(10^{\circ}\right.$ ) after selective embolization of the posterior/lateral PA (dotted arrow). Note the retrograde filling of the anterior/lateral PA (straight arrow) through intraprostatic interpedicular anastomoses that enabled retrograde embolization of the anterior/ lateral PA. (i) Pelvic CT angiography with coronal MIP reformat shows the left middle rectal artery (arrow). (j) DSA of the left middle rectal artery (arrow) with same-side anterior oblique projection $\left(35^{\circ}\right)$ and caudal/cranial angulation $\left(10^{\circ}\right)$. (k) DSA of the left middle rectal artery in neutral position with rectal wall opacification, retrograde filling of the inferior mesenteric artery (IMA), and a prominent anal blush (arrow). (Available in color online at www.jvir.org.) 

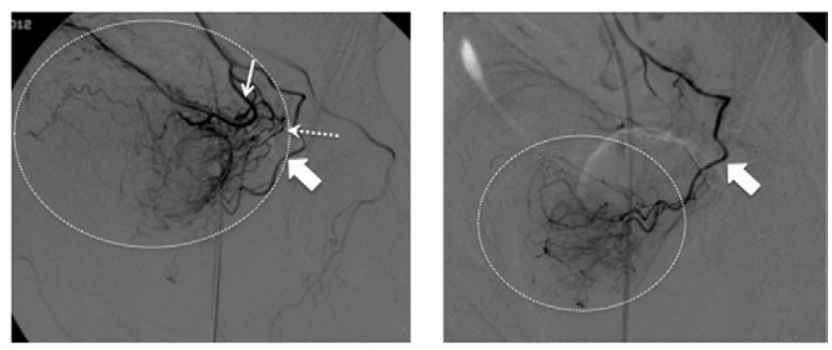

a.

b.

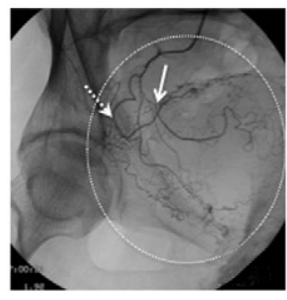

c.

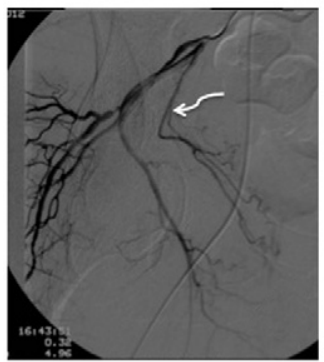

f.

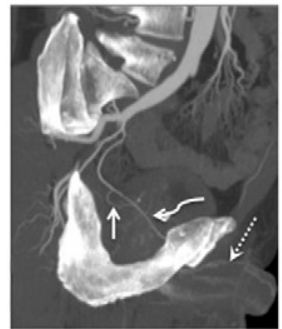

i

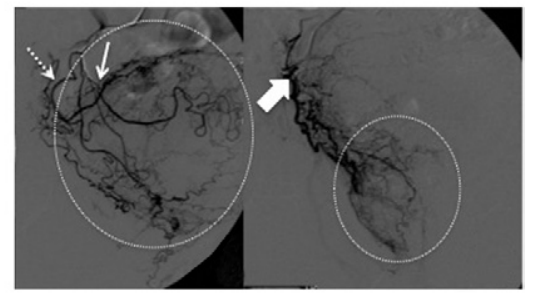

d.

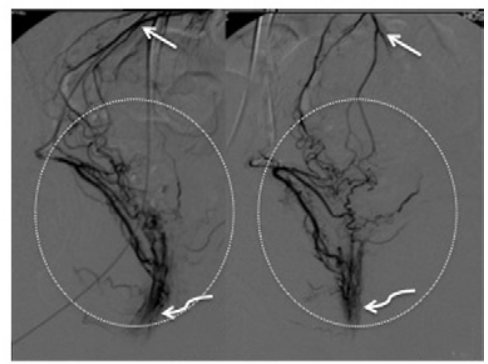

g.

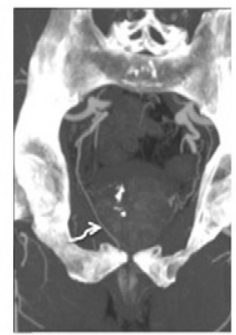

j. h.

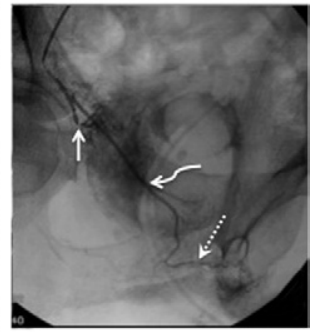

k.

Figure 8. Illustrations of a potential pitfall of PAE, namely, neighboring arteries that may be confused with PAs: (a-e) vesical arteries and PAs, (f-h) rectal arteries, and (i-k) accessory pudendal arteries. (a) Left superior vesical artery (arrow) DSA with same-side anterior oblique projection $\left(35^{\circ}\right)$ and caudal/ cranial angulation $\left(10^{\circ}\right)$ shows diffuse bladder opacification (inside circle). The dotted arrow marks the inferior vesical artery and the solid arrow marks PA. (b) After selective PA (solid arrow) DSA with same-side anterior oblique projection (35\%) and caudal/cranial angulation $\left(10^{\circ}\right)$, prostate opacification is seen (inside circle). (c, d) Diffuse bladder opacification (inside circle) after selective DSA with same-side anterior oblique projection $\left(35^{\circ}\right)$ and caudal/cranial angulation $\left(10^{\circ}\right)$ of the superior (arrow) and inferior (dotted arrow) right vesical arteries arising from a common trunk. (e) Selective right-side PA (solid arrow) DSA with same-side anterior oblique projection $\left(35^{\circ}\right)$ and cau$\mathrm{dal} / \mathrm{cranial}$ angulation $\left(10^{\circ}\right)$ shows prostate opacification (inside circle). (f) DSA of the right middle rectal artery (curved arrow) with same-side anterior oblique projection $\left(35^{\circ}\right)$ and caudal/ cranial angulation $\left(10^{\circ}\right)$. (g) Selective DSA with same-side anterior oblique projection $\left(35^{\circ}\right)$ and caudal/cranial angulation $\left(10^{\circ}\right)$ of the right middle rectal artery shows anal blush (curved arrow) and retrograde filling of the IMA (arrow). Diffuse rectal

90 minutes and a mean fluoroscopic time of approximately 30 minutes. However, even with preinterventional image guidance, as many as $3 \%$ of cases result in technical failure (ie, no embolization on either pelvic side), and as many as $8 \%$ of patients may be amenable to only unilateral embolization. From our experience, it is very difficult to predict with complete accuracy if bilateral embolization will be technically feasible based on the $\mathrm{CT}$ angiography findings. Although this is not proven in the present study, we believe PA diameter is not the most important parameter for selective catheterization; the angle of origin and the presence of atherosclerotic plaques near the ostium of the PAs are more important factors that may hamper selective catheterization, especially when the PAs arise from vesical or prostatorectal arteries. Tortuosity of the iliac arteries and PAs may also lead to technical difficulties.

Surprisingly, prostate volume did not correlate with PA size in the present study. Patients with only one PA on each pelvic side had larger PAs than those with more than one PA on each pelvic side, but no other associations were found.

Two independent PAs were found in $43 \%$ of pelvic sides $(n=64)$, which is a higher incidence than previously reported rates $(7,8,10,16)$. From our experience, the increasing awareness of the possibility of more than one PA on each pelvic side raised the suspicion for small branches that may have been initially overlooked. In the present study, we showed that the prostate has a dual blood supply, as described by Bouissou and Talazac (9), with two PAs that arose from a solitary PA or that arose independently. We opted to use the term anterior/lateral PAs instead of "cranial" PAs (9) because they penetrated the prostatic capsule in the anterior/lateral quadrants, whereas we used the term posterior/lateral PAs instead of "caudal" PAs (9) because they penetrated the prostatic capsule in the posterior/lateral quadrants. As described by Bouissou and Talazac (9), when these two PAs arose independently, the anterior/lateral PA had a more proximal origin in close proximity to the vesical arteries, whereas the posterior/ lateral PA had a more distal origin close to rectal branches.

opacification is seen inside the circle. (h) Selective DSA in neutral position of the right middle rectal artery shows anal blush (curved arrow) and retrograde filling of the IMA (arrow). Diffuse rectal opacification is seen inside the circle. (i) Pelvic CT angiography with sagittal MIP reformat of the right pelvic side. The curved arrow marks the right accessory pudendal artery that courses near the prostate and ends as the dorsal artery of the penis (dotted arrow). The straight arrow marks the PA arising from the accessory pudendal artery. (j) Pelvic CT angiography with coronal MIP reformat. The curved arrow marks the right accessory pudendal artery. (k) DSA of right accessory pudendal artery (curved arrow) performed with same-side anterior oblique projection $\left(35^{\circ}\right)$ and caudal/cranial angulation $\left(10^{\circ}\right)$. The dotted arrow marks the dorsal artery of the penis; the straight arrow marks the PA. 


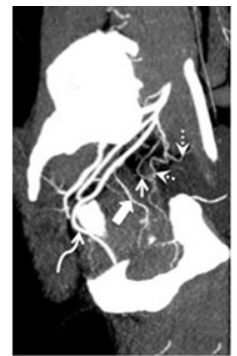

a.

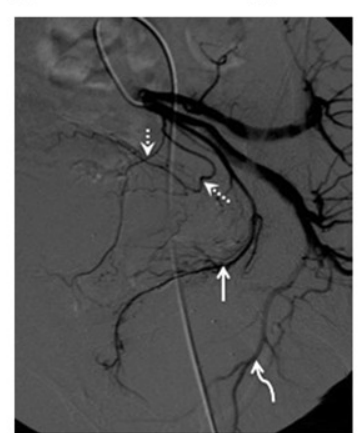

f.

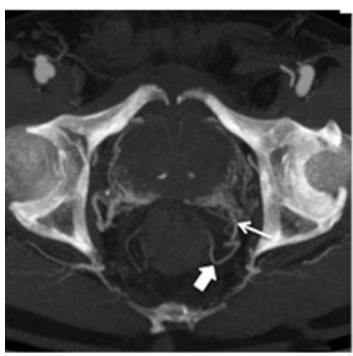

j.

b.
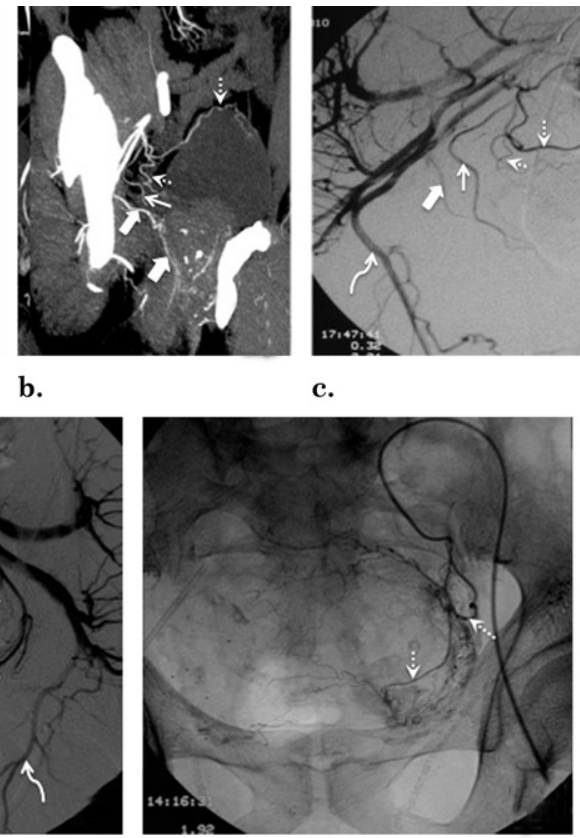

g.

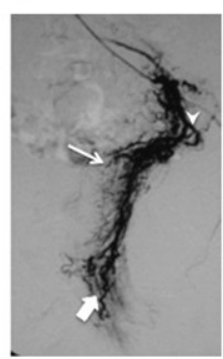

k.

c.

1.
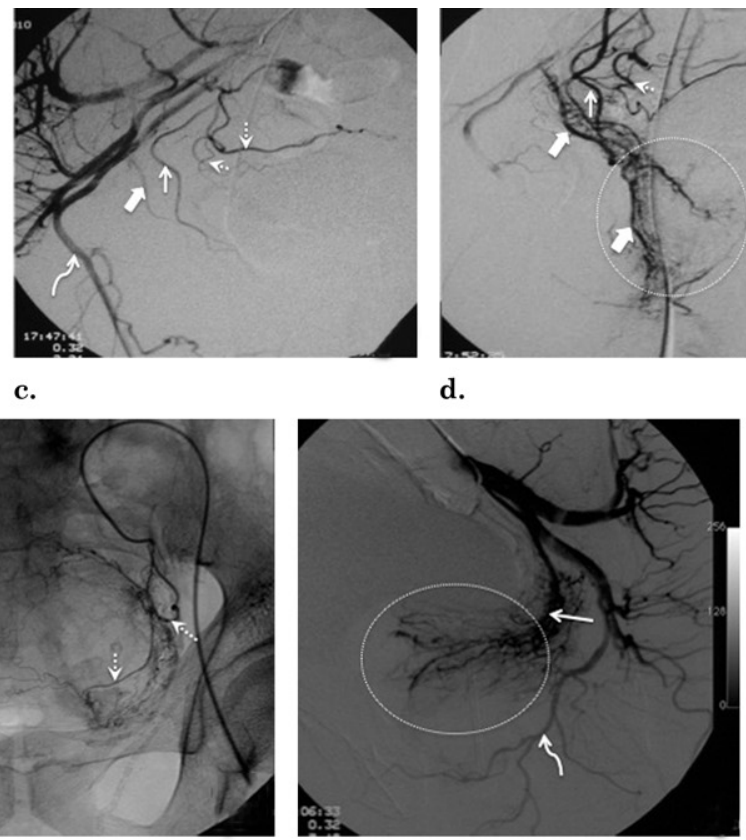

h.
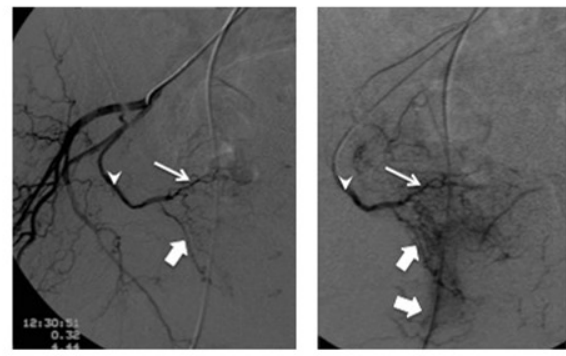

m.
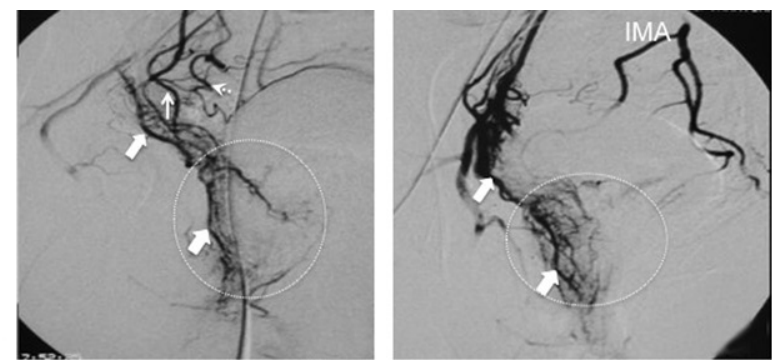

e.

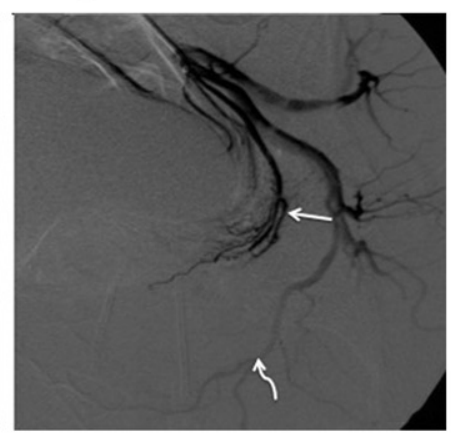

i.

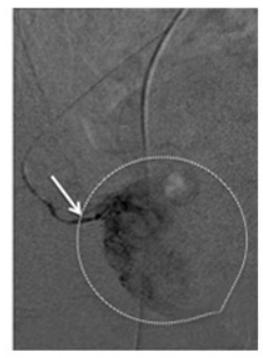

n.

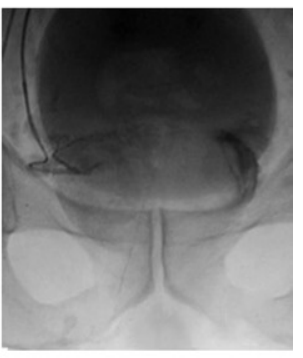

o.

Figure 9. Vesical, rectal, and prostatic arteries. (a) Pelvic CT angiography with sagittal MIP reformat of the right pelvic side. The anterior/lateral PA (straight arrow) arises from a short common trunk with the vesical arteries (dotted arrows). There is a common prostatorectal trunk (thick arrow) supplying the posterior/lateral PA and running downward into the perineum adjacent to the prostatic capsule (middle rectal artery). The curved arrow marks the internal pudendal artery. (b) Pelvic CT angiography with sagittal MIP reformat of the right pelvic side. The straight arrow marks the anterior/lateral PA, dotted arrows mark vesical arteries, and thick arrows mark the common prostatorectal trunk. (c) DSA of the right internal iliac artery performed with same-side anterior oblique projection $\left(35^{\circ}\right)$ and caudal/cranial angulation $\left(10^{\circ}\right)$. The straight arrow marks the anterior/lateral PA, dotted arrows mark the vesical arteries, the thick arrow marks the common prostatorectal trunk, and the curved arrow marks the internal pudendal artery. (d) DSA after selective catheterization of the common prostatorectal trunk (thick arrows) with same-side anterior oblique projection $\left(35^{\circ}\right)$ and caudal/cranial angulation $\left(10^{\circ}\right)$. There is diffuse prostatic and rectal opacification with anastomoses and retrograde filling of the anterior/lateral prostatic arterial pedicle (straight arrow) and vesical arteries (dotted arrow) and of the IMA, rendering embolization unsafe. Prostate gland opacification is seen inside the dotted circle. (e) DSA after selective catheterization of the common prostatorectal trunk (thick arrows) in neutral position. Prostate gland opacification is seen inside the dotted circle, and there is retrograde filling of the IMA. (f-i) Vesical and prostatic arteries in another patient (left pelvic side). (f) DSA of the internal iliac artery performed with same-side anterior oblique projection $\left(35^{\circ}\right)$ and caudal/cranial angulation $\left(10^{\circ}\right)$. The PA (straight arrow) arises from the vesical arteries (dotted arrows). The curved arrow marks the internal pudendal artery. (g) Selective DSA of the vesical arteries (dotted arrows) in neutral position shows diffuse bladder wall opacification. (h) Selective DSA with same-side anterior oblique projection $\left(35^{\circ}\right)$ and caudal/cranial angulation $\left(10^{\circ}\right)$ of the PA (straight arrow) before PAE. The curved arrow marks the internal pudendal artery. Prostate gland opacification is seen inside the dotted circle. (i) Control angiogram with same-side anterior oblique projection $\left(35^{\circ}\right)$ and caudal/cranial angulation $\left(10^{\circ}\right)$ after PAE. The straight arrow marks the PA and the curved arrow marks the internal pudendal artery. (j) Axial CT angiography scan shows a common prostatorectal trunk on the left side bifurcating into the posterior/lateral PA (straight arrow) and the middle rectal artery (thick arrow). (k) Selective DSA with same-side anterior oblique projection $\left(35^{\circ}\right)$ and caudal/cranial angulation $\left(10^{\circ}\right)$ of the common prostatorectal trunk on the left side (arrowhead) shows diffuse rectal/anal opacification (thick arrow) and prostatic opacification (straight arrow), which was difficult to isolate, rendering embolization unsafe. (I) DSA of the right pelvic side with same-side anterior oblique projection $\left(35^{\circ}\right)$ and caudal/cranial angulation $\left(10^{\circ}\right)$ shows a common prostatorectal trunk (arrowhead) that bifurcates into the middle rectal artery (thick arrow) and the PA (straight arrow). (m) DSA with same-side anterior oblique projection $\left(35^{\circ}\right)$ and caudal/cranial angulation $\left(10^{\circ}\right)$ of the common prostatorectal trunk (arrowhead) that bifurcates into the middle rectal artery (thick arrows) and the PA (straight arrow). (n) DSA with same-side anterior oblique projection ( $\left.35^{\circ}\right)$ and caudal/cranial angulation $\left(10^{\circ}\right)$ of the PA (arrow) shows prostate gland opacification (inside dotted circle). In these situations, it is possible to isolate the prostatic circulation from the rectal circulation by advancing the microcatheter distally. (o) DSA in neutral position shows prostate gland opacification after embolization. 


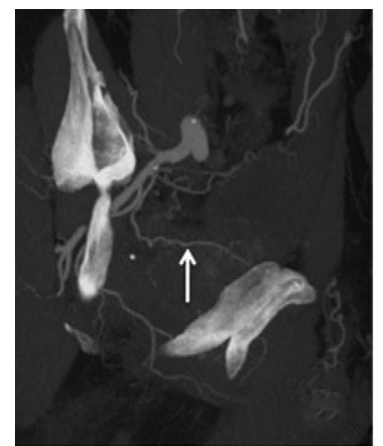

a.

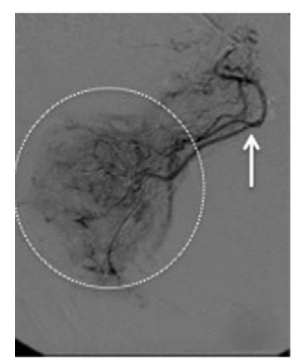

d.

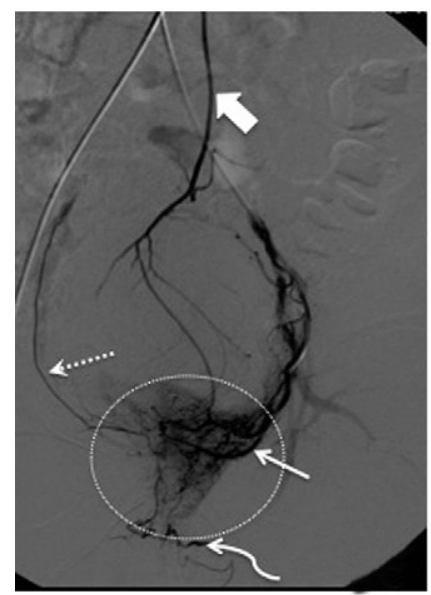

h.

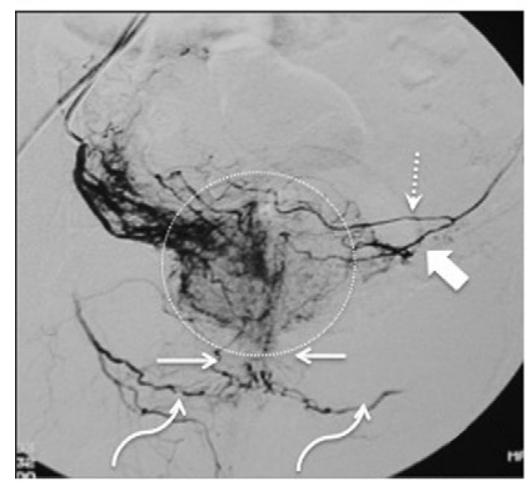

k.

b.

e.

i.
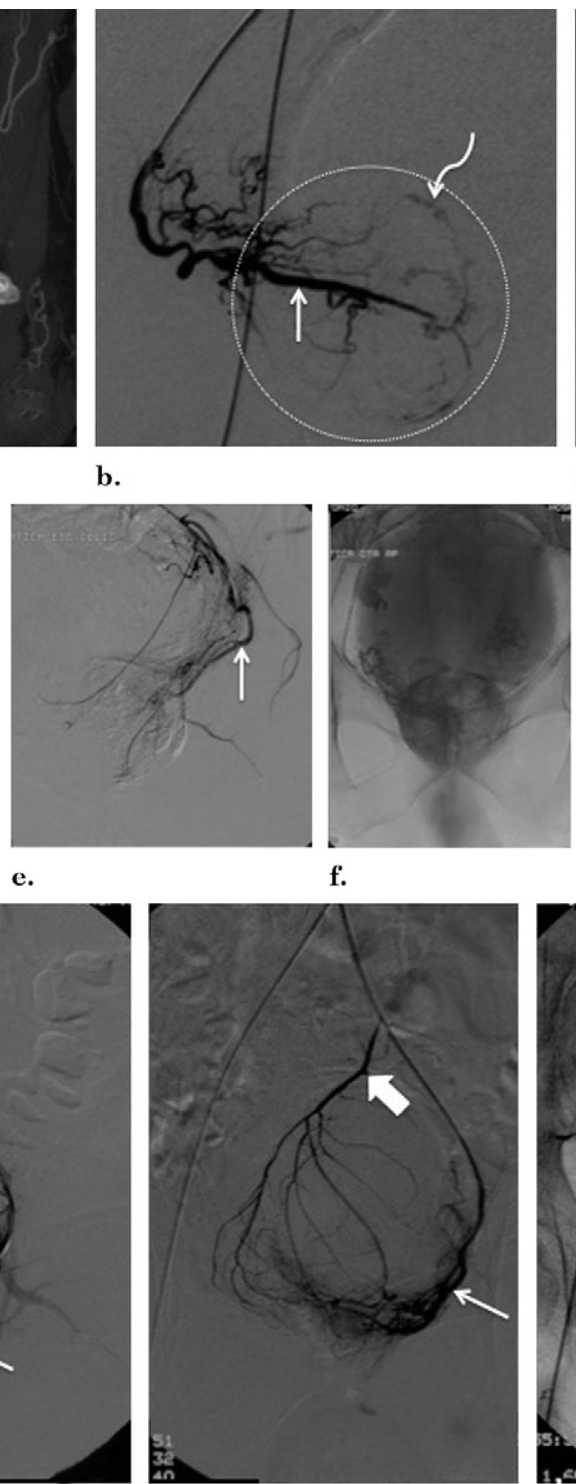

f.

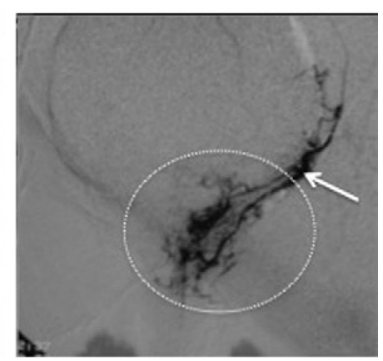

g.

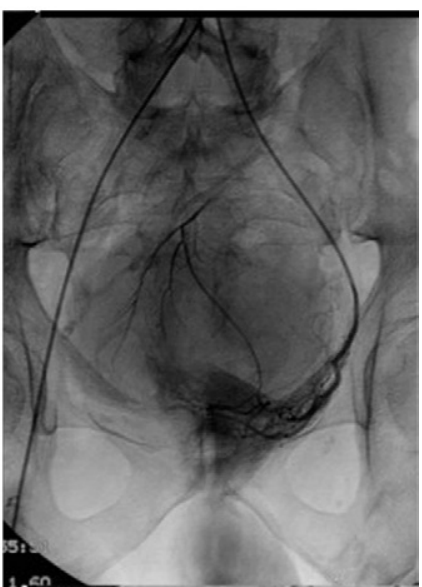

j.

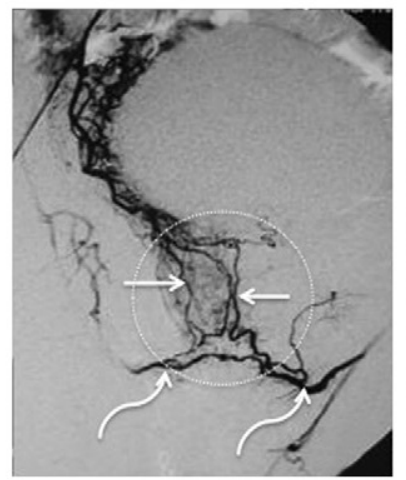

l.

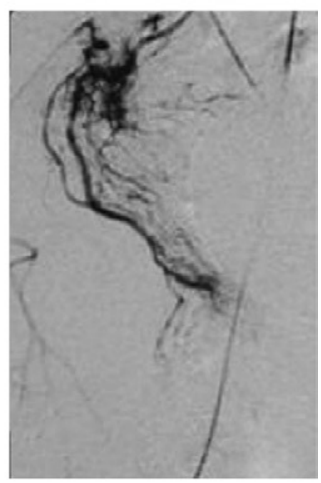

m.

Figure 10. DSA findings of PAs and arterial anastomoses. (a) Sagittal MIP CT angiography shows a right-side PA (straight arrow). (b, c) Selective DSA with same-side anterior oblique projection $\left(35^{\circ}\right)$ and caudal/cranial angulation $\left(10^{\circ}\right)$ of the right-side PA (straight arrow). The dotted circle marks prostate gland opacification, and a corkscrew pattern is shown (curved arrow). (d) Selective DSA with same-side anterior oblique projection $\left(35^{\circ}\right.$ ) and caudal/cranial angulation (10 $)$ of a left-side PA (straight arrow) before PAE. The dotted circle marks prostate gland opacification. (e) Selective DSA with same-side anterior oblique projection $\left(35^{\circ}\right)$ and caudal/cranial angulation $\left(10^{\circ}\right)$ of a left-side PA (straight arrow) after PAE. (f) DSA in neutral position shows prostate gland opacification after bilateral PAE. (g) Selective DSA (neutral position) of a left-side PA (straight arrow) shows a more diffuse and heterogeneous pattern of prostatic glandular opacification (dotted circle). (h) DSA of the left-side PA (straight arrow) in neutral position before PAE shows diffuse 


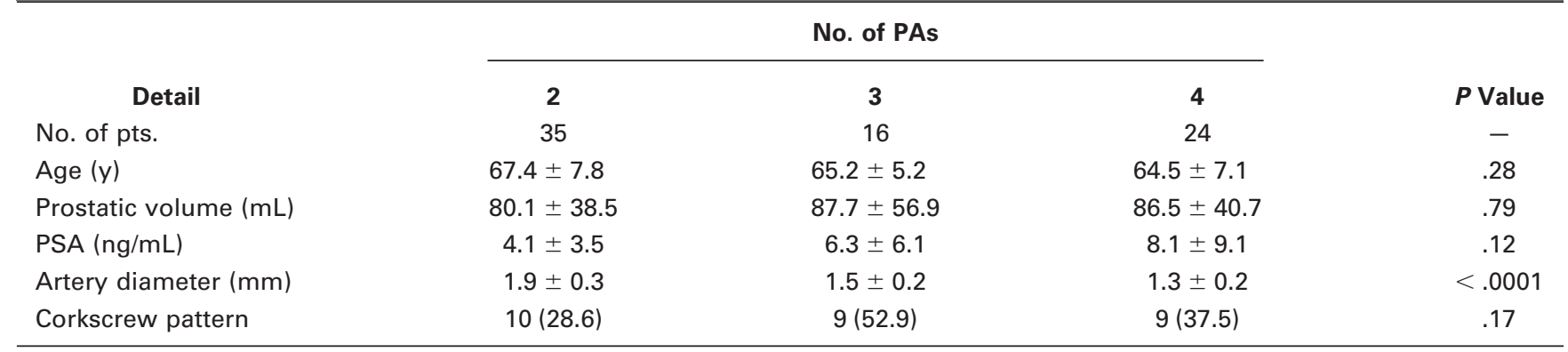

$\mathrm{PA}=$ prostatic artery, PSA $=$ prostate-specific antigen, $\mathrm{SD}=$ standard deviation.

Values presented as mean \pm SD where appropriate. Values in parentheses are percentages.

Table 3. Patient Data According to Presence or Absence of the Corkscrew Pattern

\begin{tabular}{lccc} 
& \multicolumn{2}{c}{ Corkscrew Pattern } & \\
\cline { 2 - 3 } \multicolumn{1}{c}{ Detail } & Present & Absent & P Value \\
No. of pts. & 28 & 47 & - \\
Age (y) & $66.5 \pm 7.3$ & $65.7 \pm 7.1$ & .63 \\
Prostatic volume $(\mathrm{mL})$ & $111.9 \pm 52.8$ & $67.0 \pm 24.3$ & $<.0001$ \\
PSA (ng/mL) & $8.1 \pm 8.5$ & $4.3 \pm 4.5$ & .004 \\
Artery diameter $(\mathrm{mm})$ & $1.6 \pm 0.3$ & $1.6 \pm 0.4$ & .58 \\
\hline
\end{tabular}

$\mathrm{PSA}=$ prostate-specific antigen, $\mathrm{SD}=$ standard deviation. Values presented as mean \pm SD where appropriate.

The vesical and rectal arteries may represent pitfalls to PAE, and may be confused with PAs. Here we showed DSA findings that differentiate PAs from vesical or rectal arteries.

The anterior/lateral PA was shown to be the main supply to the central gland, and therefore should probably be the preferred artery to embolize when two independent PAs are present. Embolization of one of the two PAs might be enough because of the intraprostatic interpedicular anastomoses that may be found, as previously described by Bouissou and Talazac (9). When only one PA is found, we usually leave the catheter tip before its bifurcation to allow embolization of both PAs. Further studies in patients with two independent PAs are needed to compare embolization of both PAs versus only one PA.

In the present study, findings of PA origin were somewhat different from previously published results in cadaveric specimens $(7,8,10,17)$. These differences may be explained by distinct anatomic terminology and techniques used in cadaveric versus in vivo studies. It was stated (7) that, in most individuals, $41.5 \%-74.3 \%$ of the prostate gland is supplied by a common prostate/vesical arterial trunk (also named the inferior vesical artery) that gives rise to the inferior vesical artery and the PA. However, Clegg (7) reported that the so-called inferior vesical artery might terminate supplying the prostate without any branches to the bladder. We also found that many of the arteries supplying the prostate had no relation to the vesical arteries, and therefore decided to replace the term "inferior vesical artery" with the term "PA," which may or may not have inferior vesical branches.

Significant anastomoses may be identified on DSA in as many as $60 \%$ of individuals, usually to the perineal, middle rectal, or anal branches of the internal pudendal artery, as previously described (7). From our experience, in the presence of small-sized anastomoses through capsular branches, a slow and controlled infusion may avoid distal embolization with nontarget ischemia to the bladder, rectum, anus, or corpus cavernosum. Large (2-3 $\mathrm{mm})$ precapsular branches may represent lateral accessory pudendal arteries. In cases in which this artery ends as the dorsal artery of the penis - and is therefore solely responsible for arterial blood supply to the corpora cavernosa, as found in $3.3 \%$ of pelvic sides in the present series - the best option for embolization is to selectively embolize all prostatic branches, without compromising

prostatic glandular opacification (inside circle) with anastomoses to rectal branches, with retrograde filling of the IMA (thick arrow) and anastomoses to the contralateral PA (dotted arrow) and internal pudendal artery (curved arrow) through small postcapsular branches. (i, j) DSA of the left-side PA (straight arrow) in neutral position after PAE. The thick arrow marks the IMA. (k) Selective DSA of the right-side PA in neutral position shows small pericapsular and postcapsular anastomoses (straight arrows) to the internal pudendal artery (curved arrows) and anastomoses to contralateral PA (thick arrow) and inferior vesical artery (dotted arrow). Prostatic glandular opacification is seen inside the circle. (I) DSA of the right-side PA in neutral position before PAE shows multiple arterial anastomoses through larger precapsular branches that course alongside the anterior and lateral aspect of the prostate from the base to the apex (straight arrows), terminating in the perineum-also called lateral accessory pudendal arteries-with anastomoses to the internal pudendal artery (curved arrows). Prostatic glandular opacification is seen inside the circle. (m) DSA of the right-side PA in neutral position after PAE. 
this artery. If this artery terminates as an anastomotic arch, as found in $20 \%$ of pelvic sides in the present series, with the termination of the internal pudendal artery, another option is to use $2-3-\mathrm{mm}$ microcoils or Gelfoam torpedoes to exclude this anastomosis and then embolize the entire artery with particles. Accessory pudendal arteries may represent pitfalls to PAE and may be confused with PAs; the main DSA findings in the present study may help to differentiate them.

Anastomoses with contralateral PAs may be present in as many as $20 \%$ of individuals, which might explain why some patients have good clinical results even when only unilateral embolization is achieved (6). Further studies comparing clinical outcomes between patients with unilateral and bilateral PAE are needed to assess this issue.

The limitations of the present study include the absence of cone-beam CT during selective DSA of the PAs, which may be helpful to certify correct catheter position before embolization, especially in the initial learning curve or in doubtful cases; however, we do not believe routine use is necessary if $\mathrm{CT}$ angiography has been performed and the operators have experience in PAE. Other limitations relate to the classification of the PA origin that may vary depending on the anatomic classifications used. Also, small anastomoses may go undetected with conventional angiography, and we did not use wedged catheter DSA runs. Future studies addressing anastomosis evaluation with the use of intraprocedural cone-beam CT or with cadavers may also help with this subject. It would be very important to identify the diameter of the small anastomoses to guide the choice of embolic material size to avoid extraprostatic embolization. In the present study no evaluation was performed of how the knowledge gained from CT angiography affected the embolization technique.

PAE has been shown to be a promising new minimally invasive therapeutic option for BPH; however, to perform the procedure in a safe manner without nontarget embolization to periprostatic tissues, precise knowledge of the PA anatomy and imaging findings is required.
In this work, we describe the vascular anatomy of the prostate by using CT angiography and DSA, focusing on the most relevant aspects for PAE.

\section{REFERENCES}

1. Mauro MA. Can hyperplastic prostate follow uterine fibroids and be managed with transcatheter arterial embolization? Radiology 2008; 246:657-658.

2. Sun F, Sánchez FM, Crisóstomo V, et al. Benign prostatic hyperplasia: transcatheter arterial embolization as potential treatment-preliminary study in pigs. Radiology 2008; 246:783-789.

3. Jeon GS, Won JH, Lee BM, et al. The effect of transarterial prostate embolization in hormone-induced benign prostatic hyperplasia in dogs: a pilot study. J Vasc Interv Radiol 2009; 20:384-390

4. DeMeritt JS, Elmasri FF, Esposito MP, Rosenberg GS. Relief of benign prostatic hyperplasia-related bladder outlet obstruction after transarterial polyvinyl alcohol prostate embolization. J Vasc Interv Radiol 2000; 11:767-770.

5. Carnevale FC, Antunes AA, da Motta Leal Filho JM, et al. Prostatic artery embolization as a primary treatment for benign prostatic hyperplasia: preliminary results in two patients. Cardiovasc Intervent Radiol 2010; 33:355-361.

6. Pisco JM, Pinheiro LC, Bilhim T, Duarte M, Mendes JR, Oliveira AG. Prostatic arterial embolization to treat benign prostatic hyperplasia. $J$ Vasc Interv Radiol 2011; 22:11-19.

7. Clegg EJ. The vascular arrangements within the human prostate gland. $\mathrm{Br} J$ Urol 1956: 28:428-435.

8. Clegg EJ. The arterial supply of the human prostate and seminal vesicles. J Anat 1955; 89:209-216.

9. Bouissou H, Talazac A. Arterial vascularization of the normal and the pathological prostate. Ann Anat Pathol 1959; 4:63-79.

10. Ambrósio JD, De Almeida JS, De Souza A. Origin of prostatic arteries in man. Rev Paul Med 1980; 96:52-55.

11. Slojewski M, Czerwinski F, Sikorski A. Microangiographic imaging of the prostate. BJU Int 2002; 89:776-778.

12. Duclos JM, Chanzy M, Alexandre JH. Prostatic vascularisation. Arch Anat Pathol 1972; 20:355-358.

13. Ambrósio JD, De Almeida JS, De Souza A. Apparent penetration of the arteries into the prostatic capsule in man. Rev Paul Med 1980; 95:98100

14. Yamaki K, Saga T, Doi Y, et al. A statistical study of the branching of the human internal iliac artery. Kurume Med J 1998; 45:333-340.

15. Bilhim T, Casal D, Furtado A, Pais D, O'Neill JE, Pisco JM. Branching patterns of the male internal iliac artery: imaging findings. Surg Radiol Anat 2011: 33:151-159.

16. Bilhim T, Pisco JM, Furtado A, et al. Prostatic arterial supply: demonstration by multirow detector angio CT and catheter angiography. Eur Radiol 2011; 21:1119-1126.

17. Chen $X Q$. Anatomic study of the arteries and nervous plexus of the prostate and their clinical significance. Zhonghua Wai Ke Za Zhi 1989; 27:373-375. 